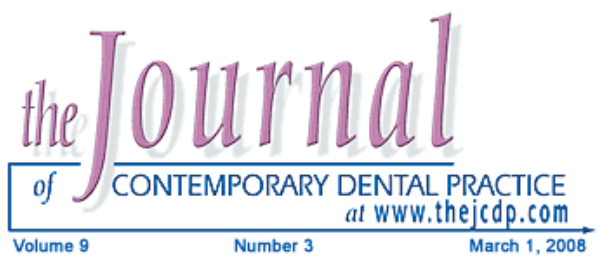

\title{
Treatment of a Patient with Oligodontia: A Case Report
}

\author{
Nursel Akkaya, DDS, PhD; Arlin Kiremitçi, DDS, PhD;
}

Ozden Kansu, DDS, PhD

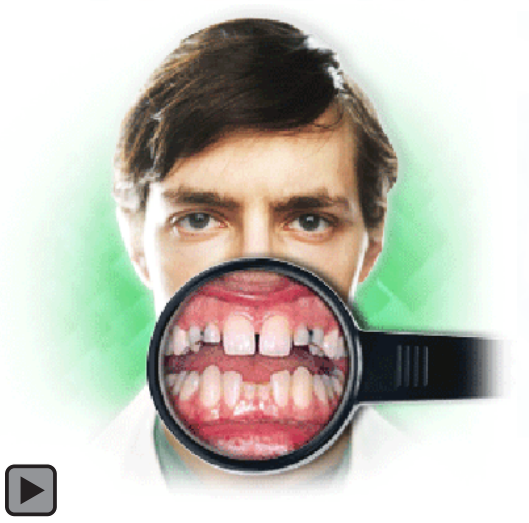

Abstract

Aim: The aim of this report is to describe the management of a 16-year-old patient with oligodontia including six permanent teeth.

Background: Oligodontia is agenesis of six teeth or more, excluding third molars. The etiology of congenital absence of teeth is believed to be involved in heredity or developmental anomalies. It can be isolated or as part of a syndrome. There are a number of options available to restore space generated by missing teeth. Dental treatment can vary depending on the severity of the disease and generally requires a multidisciplinary approach. Treatment options include orthodontic therapy, implants, adhesive techniques, and removable prostheses.

Report: A 16-year-old male patient with oligodontia affecting six permanent teeth received conservative care that met his and his parent's expectations. The existing primary teeth were restored to resemble permanent teeth in order to achieve a favorable esthetic result using direct composite resin. The restorative treatment was provided for the psychosocial comfort of the young patient. The loss of teeth in young patients can cause esthetic, functional, and psychological problems particularly if the teeth of the anterior region are involved.

Summary: Adhesive techniques and new restorative materials represent current options in the management of the dental rehabilitation of young patients with oligodontia.

Keywords: Tooth abnormalities, oligodontia, tooth agenesis, taurodontism

Citation: Akkaya N, Kiremitçi A, Kansu Ö. Treatment of a Patient with Oligodontia: A Case Report. J Contemp Dent Pract 2008 March; (9)3:121-127.

(c) Seer Publishing 


\section{Introduction}

Oligodontia is defined as the developmental absence of six teeth or more, excluding third molars. ${ }^{1,2}$ It can be isolated (Oligodontia-I) or as a part of a syndrome (Oligodontia-S) such as in ectodermal dysplasia. ${ }^{3}$ Oligodontia in the permanent dentition occurs in $0.3 \%$ of the population. ${ }^{1}$ Congenital absence of teeth most commonly occurs with the maxillary lateral incisors, second premolars, and mandibular central incisors. It may be either unilateral or bilateral. ${ }^{4}$ The absence of maxillary central incisors, maxillary and mandibular canines, or first molars is rare and mostly occurs in patients with oligodontia. ${ }^{1}$ Radiography is used for confirmation of congenitally missing teeth. ${ }^{4}$

Existing primary teeth in patients with oligodontia should be restored because of esthetic, phonetic, and nutritional problems. ${ }^{5}$ Treatment can include space closure or space opening before restorative procedures with orthodontic therapy, removable or fixed partial dentures, implantretained prosthesis, or a combination of these treatment strategies. ${ }^{1}$

This report describes the management of a patient with oligodontia including bilateral missing maxillary lateral incisors, canines and mandibular central incisors together with taurodontism of the maxillary and mandibular second molars as well as the patient's restorative treatment.

\section{Case Report}

\section{Diagnosis}

A 16-year-old male was referred to the Department of Oral Diagnosis and Radiology with a chief complaint of missing permanent teeth and an unaesthetic appearance. His medical history was noncontributory. There was no history of previous extractions. The family history revealed his father, his sister, and brother were also afflicted with hypodontia. Extraoral examination revealed the patient had no abnormalities so no additional laboratory tests were needed to investigate any systemic condition that may have contributed to the problem.

Intraoral examination revealed the presence of maxillary left and right primary lateral incisors, canines, and mandibular primary central incisors.
The teeth were not mobile. There was evidence of caries on the distal aspects of the primary lateral incisors as well as on the mesial and distal aspects of primary canine teeth which were asymptomatic (Figure 1). The third molars were not erupted.

A panoramic radiograph confirmed the bilateral absence of all permanent teeth which were not present clinically (Figure 2). There was evidence of minimal root resorption of the primary canines, and the maxillary and mandibular second molars were taurodont. The other permanent teeth were normal. A radiopaque appearance was detected in the periradicular region of maxillary left premolar.

This region was examined on periapical radiograph, and it was diagnosed as osteosclerosis or enostosis (Figure 3).

Radiographically all third molars were unerupted and there was evidence of caries on distal aspect of mandibular left first molar.

\section{Treatment}

During treatment planning the patient's age was considered along with oral hygiene status, socioeconomic background, and his treatment expectations. The patient and his parents were informed about the existing condition and the objectives of treatment. Dental treatment consisted of preventive and restorative phases. The preventive phase was done first which included a professional cleaning to remove dental

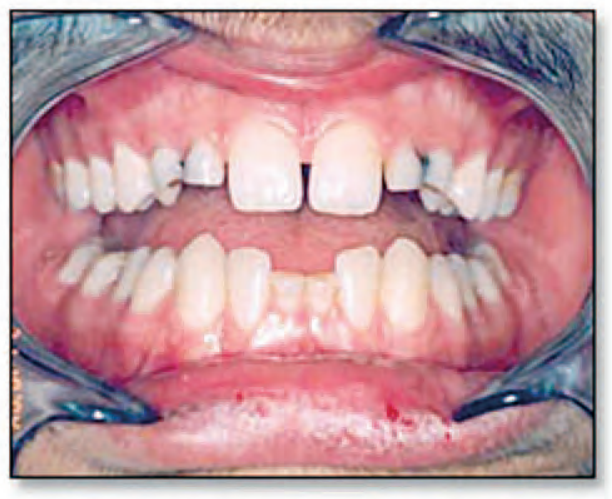

Figure 1. Intraoral examination showing bilaterally missing permanent maxillary lateral incisors, canines, and mandibular central incisors. 


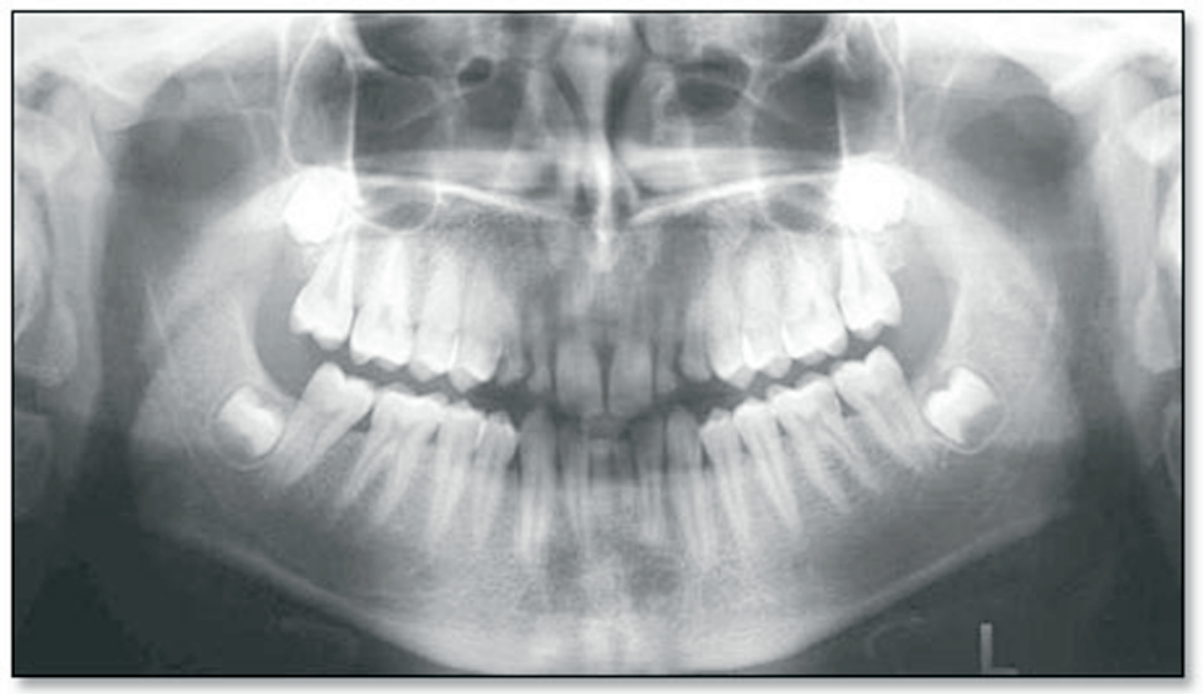

Figure 2. Panoramic radiograph showing agenesis of six permanent teeth and taurodontism of second molars.

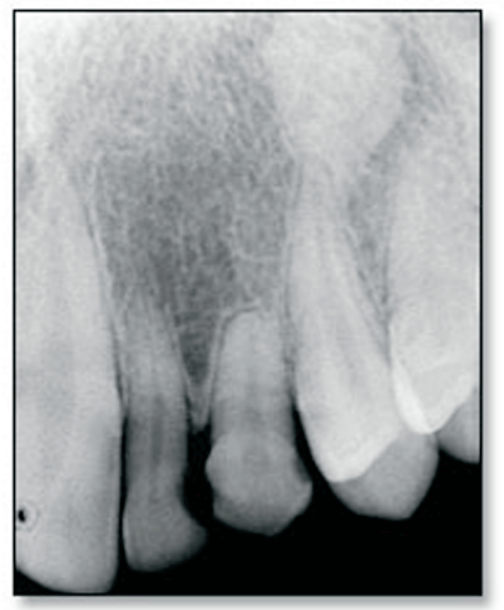

Figure 3. Periapical radiograph showing sclerotic changes of periradicular region of maxillary left premolar.

stain and oral hygiene instructions. Restorative treatment using direct composite resin restorations was the most appropriate approach for this patient since the maxillary and mandibular anterior teeth would continue to undergo passive eruption for more than 20 years. The primary teeth were restored with Miris hybrid composite resin (Coltène Whaledent, Altstätten, Switzerland) to resemble the permanent teeth (Figure 4). One year later, the patient declined a follow-up visit because he stated he no complaints about his teeth at that time.

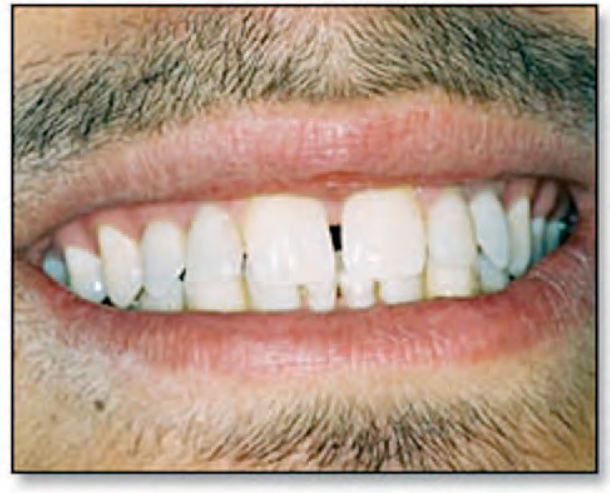

Figure 4. After the restorative treatment of primary teeth by using direct composite resin.

\section{Discussion}

In this case the absence of six permanent teeth together with taurodontism of second molars was observed. Seow and $\mathrm{Lai}^{6}$ reported $34.8 \%$ of patients with hypodontia had at least one mandibular permanent molar with taurodontism.

The etiology of congenital absence of teeth is believed to be involved in heredity or developmental anomalies. ${ }^{2}$ Graber $^{7}$ claimed the congenital absence of teeth was largely due to hereditary factors. The family history has to be considered in such cases as was done in the present case. It is also important to determine whether oligodontia is related to a syndrome or not. Patients with oligodontia as a part of a 
syndrome may have abnormalities in other parts of the body, such as the skin, ears, eyes, and skeleton. ${ }^{2}$ The patient in the present case had no problems associated to these symptoms.

Panoramic radiography is a useful diagnostic tool for the diagnosis of congenital missing teeth. These provide a global view of the jaws not only for diagnosing oligodontia but also for evaluating other anomalies of the teeth such as morphologic alterations, variations of tooth size, or tooth absence. Avcu et al. ${ }^{8}$ recommended a panoramic radiographic examination when a tooth was missing because it might be an ectopic impaction. In the present case a panoramic radiograph revealed no unerupted teeth except for third molars. The radiograph was essential for the detection of taurodontism of the maxillary and mandibular second molars while evaluating the image for possible abnormalities of the other teeth.

Treatment of patients with oligodontia generally requires a multidisciplinary approach. Some patients may require prerestorative orthodontics. Restoration with a removable partial denture, conventional fixed partial denture, an implantretained prosthesis and adhesive restorative techniques, or a combination of these therapies are the treatment options. ${ }^{9}$ A number of factors must be taken into account for treatment planning. The age of the patient is the most important factor during treatment planning. Other conditions that must be evaluated include the number and condition of retained teeth, the number of missing teeth, presence of carious teeth, condition of supporting tissues, occlusion, and the interocclusal rest space. ${ }^{1}$
The age of the patient plays a significant role in selecting direct composite as the restorative material. Volchansky et al. ${ }^{10-11}$ have reported studies suggesting the maxillary and mandibular anterior teeth continued to undergo passive eruption beyond 20 years of age and the gingival architecture and papillary height were not stable in the late teens and early adulthood. With this in mind, the teeth were restored with direct composite because the patient was 16 years-old. Prognosis of the primary teeth determines the long-term prognosis of this treatment. Another option for this patient might have been extraction of the primary teeth followed by combining orthodontic therapy and rehabilition with dental implants. However, the expectations for treatment by the patient and his parents was to achieve an esthetic result at a low cost; a longterm treatment plan was rejected. Management with direct composite was the least-expensive treatment alternative for cosmetic reconstruction. In addition, this simple and noninvasive approach provided psychosocial comfort for the young patient.

An advantage of a more conservative treatment plan, in this case prosthetic rehabilition, remains as an alternative treatment option for the future.

\section{Summary}

Adhesive techniques and new restorative materials represent current options in the management of the dental rehabilitation of young patients with oligodontia. Restorative treatment was provided for the psychosocial comfort of the young patient in this case.

\section{Refernces}

1. Dhanrajani PJ. Hypodontia: etiology, clinical features, and management. Quintessence Int 2002; 33:294-302.

2. Schalk-van der Weide Y, Beemer FA. Symtomatology of patients with oligodontia. J Oral Rehabil 1994; 21:247-261.

3. Schalk-Van Der Weide Y, Bosman F. Tooth size in relatives of individuals with oligodontia. Arch Oral Biol 1996; 41:469-472.

4. Goaz PW, White SC. Dental Anomalies. In: Goaz PW, White SC, eds. Oral Radiology Principles and Interpretation. 3rd ed. St. Louis: Mosby; 1994: 340-368.

5. Eronat N, Ertugrul F. An unusual case of hypodontia with extensive caries: A multidisciplinary treatment approach. J Clin Pediatr Dent 1991; 15:199-201.

6. Seow WK, Lai PY. Association of taurodontism with hypodontia. Pediatr Dent 1989; 11:214-219.

7. Graber LW. Congenital absence of teeth: a review with emphasis on inheritance patterns. J Am Dent Assoc 1978; 96:266-275. 
8. Avcu N, Buyukkopru D, Kansu O, Dural S. Severe hypodontia and asymptomatic bilaterally ectopic impacted teeth in the coronoid processes: A case report. Quintessence Int 2004; 35:582-583.

9. Wagenberg BD, Spitzer DA. Therapy for patient with oligodontia: case report. J Periodontol 2000; 71:510-516.

10. Volchansky A, Cleaton-Jones P. The position of the gingival margin as expressed by clinical crown height in children aged 6-16 years. J Dent 1976; 4:116-122.

11. Volchansky A, Cleaton-Jones P, Fatti LP. A 3-year longitudinal study of the position of the gingival margin in man. J Clin Periodontol 1979; 6:231-237.

\section{About the Authors}

Nursol Akkaya, DDS, PhD

Dr. Akkaya is a Research Assistant in the Department of Oral Diagnosis and Radiology of the

Faculty of Dentistry at Hacettepe University in Ankara, Turkey.

e-mall: ynursel@hacettepe.edu.tr

\section{Ariln KIremitẹl, DDS, PhD}

Dr. Kiremitgl is an Assistant Professor in the Department of Restorative Dentistry of the Faculty of Dentistry at Hacettepe University in Ankara, Turkey.

e-mail: arlin@hacettepe.edu.tr

\section{Ozdon Kansu, DDs, PhD}

Dr. Kansu is a Professor in the Department of Oral Diagnosis and Radiology of the Faculty of Dentistry at Hacettepe Unlversity in Ankara, Turkey.

e-mall: okansu@hacettepe.edu.tr 